\title{
Considerações sobre duas metodologias de análise de estabilidade e adaptabilidade ${ }^{1}$
}

\author{
Some considerations about two methodologies for stability and adaptability analysis
}

\author{
Devanir Mitsuyuki Murakami ${ }^{2}$ Antônio Américo Cardoso ${ }^{3}$ Cosme Damião Cruz $^{4}$ Nair Bizão $^{5}$
}

\section{RESUMO}

Foram avaliados 31 Híbridos comerciais de milhos na região sul do Estado de Mato Grosso, sendo 16 em safra normal 96/97 e 15 em safrinha/97, possibilitando análises de estabilidade e adaptabilidade para cada época. Cada experimento foi instalado no delineamento em blocos ao acaso com três repetições. Foram empregadas as metodologias de EBERHART \& RUSSELL (1966) (univariada), LIN \& BINNS (1988) (univariada) $e$ modificações realizadas por CARNEIRO (1998) (univariada e multivariada). Constataram-se efeitos significativos para ambientes, híbridos, interação híbridos $x$ ambientes e desvios combinados. A grande maioria dos híbridos apresentou ampla adaptabilidade e boa estabilidade segundo a metodologia de EBERHART \& RUSSELL (1966). A metodologia de LIN \& BINNS (1988) modificada por CARNEIRO (1998) foi mais discriminante do que EBERHART \& RUSSELL (1966) e por isso, mais eficiente e mais indicada para análises de estabilidade e adaptabilidade. A análise multivariada permitiu conhecer melhor a performance dos genótipos, constituindose em importante ferramenta complementar na indicação de genótipos de acordo com seu desempenho.

Palavras-chave: estabilidade, metodologia, milho.

\section{ABSTRACT}

Thirty one commercial maize hybrids were evaluated in the southern Mato Grosso State, Brazil, 16 being regular crop (1996/97) and 15 in the late of sowing (February ending and the beginnig of March, 1997) making possible analyses of stability and adaptability for each period. Each experiment was carried out in randomized block design with three repetitions. The metodologies of EBERHART \& RUSSELL (1966) (univariate analysis), LIN \& BINNS (1988) (univariate analysis) and modifications by CARNEIRO (1998) (univariate and multivariate analysis) were applied. Significant effects were verified for environment, hybrids, hybrids $x$ environment interactions and combined deviations. The majority of the hybrids showed wide adaptability and good stability according to EBERHART \& RUSSELL (1966) methodology. The methodology of LIN \& BINNS (1988) modified by CARNEIRO (1998) was more discriminant than EBERHART \& RUSSELL (1966) and because of that, more efficient and suitable for analysis of stability and adaptability. The multivariate analysis allowed to understand better the performance of the genotypes being an important complemental tool in the genotype indication in agreement with their performance.

Key words: stability, methodology, maize.

\section{INTRODUÇÃO}

A cultura do milho, no Brasil, apresenta grande dispersão geográfica, pois ele é produzido, praticamente, em todo o território nacional. Confrontase, assim, com enorme variação nas condições edafoclimáticas, infra-estrutura de produção e de mercado, além de vários outros fatores sócio-econômicos.

A indicação de cultivares considerando apenas a média geral de ensaios favorece genótipos (cultivares) que se sobressaem nos melhores ambientes e não discrimina os que se adaptam às melhores e às piores condições. $\mathrm{O}$ conhecimento do comportamento ou adaptabilidade de genótipos a

\footnotetext{
${ }^{1}$ Parte do trabalho de tese apresentado à Universidade Federal de Viçosa (UFV), Viçosa, MG, pelo primeiro autor para obtenção do título de Doctor Scientiae.

${ }^{2}$ Engenheiro Agrônomo, Mestre e Doutor em Genética e Melhoramento. Departamento de Biologia Geral, UFV, 36570-000, Viçosa, MG. E-mail: cdcruz@mail.ufv.br Autor para correspondência.

${ }^{3}$ Engenheiro Agrônomo - DS, Professor do Departamento de Fitotecnia - (UFV).

${ }^{4}$ Engenheiro Agrônomo - DS, Professor Titular do Departamento de Biologia Geral - (UFV).

${ }^{5}$ Biólogo, Mestre em genética e melhoramento, Professor do Departamento de Biologia - (UFMT).
} 
determinados ambientes é de grande importância para a avaliação do valor agronômico dos cultivares, tanto para os produtores de sementes como para os de grãos. A estabilidade da produtividade, em grande amplitude de condições ambientais, tem sido relevante para avaliar o potencial de genótipos, pois, permite a identificação de cultivares que interagem o menos possível com os ambientes. Amplos esforços devem ser feitos no sentido de identificar genótipos que possuam alta estabilidade ou com o comportamento previsível para produção em diversos ambientes.

É notório o uso da metodologia de EBERHART \& RUSSELL (1966) para estudo da adaptabilidade e estabilidade, muito provavelmente devido à sua praticidade de uso e aos resultados satisfatórios. Por outro lado, verifica-se menor uso da metodologia de LIN \& BINNS (1988) apesar desta ser mais simples e não paramétrica. Pode-se questionar o porquê dessa preferência e se há diferenças na qualidade de discriminação genotípica quanto à sua adaptabilidade e estabilidade. Segundo CRUZ \& REGAZZI (1997), a escolha de um método depende dos dados experimentais, principalmente os relacionados com o número de ambientes disponíveis, da precisão requerida e do tipo de informação desejada. Enfatizam ainda que alguns métodos são alternativos, enquanto outros são complementares, podendo ser utilizados conjuntamente.

Ométodo deEBERHART \& RUSSELL(1966)

considera a regressão de cada variedade no experimento, em relação a um índice de ambiente e uma função de desvios desta regressão. A adaptabilidade de cada variedade é dada em função do coeficiente de regressão $\left(\beta_{1 \mathrm{i}}\right)$ de modo que: quando $\beta_{1 \mathrm{i}}=1$, os genótipos são de adaptabilidade ampla ou geral; $\beta_{1 \mathrm{i}}>1$, os genótipos apresentam adaptabilidade específica a ambientes favoráveis e $\beta_{1 \mathrm{i}}<1$, os genótipos são de adaptabilidade específica a ambientes desfavoráveis. A estabilidade está relacionada com a previsibilidade de comportamento dado pelo componente de variância devido aos desvios da regressão $\left(\sigma_{\mathrm{di}}^{2}\right)$, sendo estáveis (previsíveis) quando $\sigma_{\mathrm{di}}^{2}=0$ e pouco estáveis ou instáveis (imprevisíveis) quando $\sigma_{\mathrm{di}}^{2} \neq 0$.

LIN \& BINNS (1988) definiram, como medida para estimar a performance genotípica, o quadrado médio da distância entre a média do cultivar e a resposta média máxima para todos os ambientes. Este método pondera os desvios de comportamento dos cultivares nos ambientes, ou seja, considera a estabilidade de comportamento. Além disso, leva em consideração o rendimento do genótipo e a resposta relativa a um genótipo hipotético que é uma medida de adaptabilidade. Recentemente, este método foi modificado por CARNEIRO (1998) para atender às necessidades de se identificar genótipos superiores nos grupos de ambientes favoráveis e desfavoráveis, utilizando a mesma metodologia de classificação de ambientes definidas em EBERHART \& RUSSELL (1966). Em seu trabalho, CARNEIRO (1998) enriquece ainda mais a metodologia propondo análise multivariada da adaptabilidade e estabilidade.

Este trabalho objetivou avaliar a capacidade de discriminação genotípica das metodologias de análise da adaptabilidade e estabilidade, propostas por EBERHART \& RUSSELL(1966) e LIN \& BINNS (1988), utilizando-se de híbridos comerciais de milho, considerando-se a produtividade de grãos e, simultaneamente, outros caracteres de interesse agronômico.

\section{MATERIAL E MÉTODOS}

Vários experimentos foram analisados em duas épocas, sendo: seis em safra normal 96/97 e oito em safrinha/97, na região sul do Estado de Mato Grosso, nos municípios de Campo Verde, Primavera do Leste, Rondonópolis, Serra da Petrovina e Santo Antônio do Leverger. Foram avaliados 31 híbridos comerciais de milho, sendo dezesseis nos ensaios de safra normal 1996/97 e quinze nos ensaios de safrinha/97. Utilizouse o delineamento em blocos ao acaso, com três repetições. Cada parcela foi constituída por quatro linhas de 5,20m de comprimento, espaçadas de 0,80 a $0,90 \mathrm{~m}$ entre linhas e $0,20 \mathrm{~m}$ entre plantas. Somente as duas linhas centrais foram utilizadas como parcela útil.

O Estado de Mato Grosso possui solo, predominantemente, de baixa fertilidade natural, textura variando de média a muito argilosa e bem drenados (MONTEIRO et al., 1992). O Estado possui clima classificado como Aw de Köppen e 4cTh de Gaussen, tropical, com estação seca em torno de três meses. A precipitação média anual varia entre 1250 e $1500 \mathrm{~mm}$ com trimestre mais chuvoso em dezembro, janeiro e fevereiro. A temperatura média anual é de $24^{\circ} \mathrm{C}$, sendo a máxima de $40^{\circ} \mathrm{C}$ e a mínima $0^{\circ} \mathrm{C}$ (EMBRAPA, 1993).

A análise de adaptabilidade e estabilidade foi realizada separadamente para cada época utilizando-se as metodologias de EBERHART \& RUSSELL (1966), LIN \& BINNS (1988) e modificações realizadas por CARNEIRO (1998). Dentro de cada época, foram realizadas análises univariada e multivariada. $\mathrm{Na}$ análise univariada, considerou-se apenas a produtividade de grãos $\left(\mathrm{kg} \cdot \mathrm{ha}^{-1}\right)$ e, nas multivariadas, além da produtividade de grãos, as variáveis: altura de plantas $(\mathrm{cm})$, porcentagem de plantas quebradas, porcentagem de espigas atacadas 
por lagartas, porcentagem de espigas atacadas por doenças e ocorrência de Phaeosphaeria aos 90 dias (ensaios de safra normal 96/97) e, nos ensaios de safrinha/97, além das variáveis citadas, a porcentagem de plantas acamadas.

A metodologia de EBERHART \& RUSSELL (1966) baseia-se na análise de regressão linear de cada genótipo com as variações ambientais codificadas como índice ambiental. Os coeficientes de regressão de cada genótipo em relação ao índice ambiental $\left(\beta_{1 i}\right)$ e os desvios desta regressão $\left(\sigma_{\mathrm{di}}^{2}\right)$ proporcionam estimativas de parâmetros de adaptabilidade e estabilidade, respectivamente. Para que uma variedade seja considerada "ideal", deve apresentar, além de uma média elevada, um coeficiente de regressão igual ou próximo da unidade e desvios da regressão não diferindo significativamente de zero.

Na metodologia de LIN \& BINNS (1988), o desempenho geral dos genótipos é definido como sendo o quadrado médio da distância entre a média do cultivar e a resposta média máxima para todos os locais, de modo que, genótipos com menores valores correspondem aos de melhor desempenho. Segundo esta definição, o estimador é dada por:

$P_{i g}=\frac{\sum_{j=1}^{n}\left(Y_{i j}-M_{j}\right)^{2}}{2 n}$, em que:

$\mathrm{P}_{\mathrm{ig}}=$ estimativa do parâmetro de estabilidade do cultivar $\mathrm{i}$;

$\mathrm{Y}_{\mathrm{ij}}=$ produtividade de grãos $\left(\mathrm{kg}_{\mathrm{g}} \mathrm{ha}^{-1}\right)$ do i-ésimo cultivar no j-ésimo local;

$\mathrm{M}_{\mathrm{j}}=$ resposta máxima observada entre todos os cultivares no local $\mathrm{j}$;

$\mathrm{n}=$ número de locais.

CARNEIRO (1998) decompôs a estatística

$\mathrm{P}_{\text {ig }}$ para atender à identificação de genótipos superiores nos grupos de ambientes favoráveis e desfavoráveis, utilizando a mesma metodologia de classificação de ambientes de EBERHART \& RUSSELL (1966). Nesta decomposição, os seguintes estimadores são utilizados:

$P_{i f}=\frac{\sum_{j=1}^{f}\left(Y_{i j}-M_{j}\right)^{2}}{2 f}$ e $P_{i d}=\frac{\sum_{j=1}^{d}\left(Y_{i j}-M_{j}\right)^{2}}{2 d}$

em que:

$\mathrm{P}_{\text {if }}=$ estimador de $\mathrm{P}_{\mathrm{ig}}$ para ambientes favoráveis;

$\mathrm{P}_{\mathrm{id}}=$ estimador de $\mathrm{P}_{\mathrm{ig}}$ para ambientes desfavoráveis;

$\mathrm{f}=$ número de ambientes favoráveis;

$\mathrm{d}$ = número de ambientes desfavoráveis;

$\mathrm{Y}_{\mathrm{ij}}$ e $\mathrm{M}_{\mathrm{j}}$ como definidos anteriormente.
Desta forma, a indicação de cultivares pode ser realizada para os diferentes tipos de ambientes (geral ou específica) sendo, portanto, mais completa que a metodologia proposta originalmente.

Considerando que a indicação de um cultivar ou variedade não deve ser feita baseando-se em apenas uma única variável, mesmo que esta seja produtividade, CARNEIRO (1998) propôs a decomposição multivariada do método de LIN \& BINNS (1988) alertando que este procedimento pode ser realizado desde que seja possível somar os valores dos $\mathrm{P}_{\mathrm{ig}}$ 's do genótipo, referentes a cada variável sugerindo, para tanto, a padronização

desses $\mathrm{P}_{\mathrm{ig}}$ 's, dada por: $P_{m i}=\sum_{k=1}^{v}\left[P_{i k} \frac{1}{\hat{\sigma}_{p k}}\right]$ em que $\mathrm{P}_{\mathrm{mi}}=$ estimador do parâmetro de Medida de Adaptabilidade e Estabilidade de Comportamento (MAEC), multivaridado para o cultivar i;

$\mathrm{P}_{\mathrm{ik}}=$ estimador do parâmetro MAEC para o cultivar $\mathrm{i}$ relativo à k-ésima variável;

$\sigma_{\mathrm{pk}}=$ desvio padrão dos Pi's para a k-ésima variável.

Além disso, CARNEIRO (1998) considerou um fator de multiplicação dado pelo inverso dos pesos atribuídos a cada variável a fim de balancear a importância de cada variável. Assim, o estimador do parâmetro MAEC fica:

$P_{m i}=\sum_{k=1}^{v}\left[P_{i k} \frac{1}{\hat{\sigma}_{p k}}\right] \times \frac{1}{p_{k}}$

onde: $\mathrm{p}_{\mathrm{k}}$ representa o peso atribuído pelo pesquisador à variável $\mathrm{k}$.

O autor estabelece, ainda, o genótipo hipotético ideal definido de acordo com o modelo proposto por CRUZ et al. (1989), dado por: $Y_{m j}=b_{o m}+b_{1 m} I_{j}+b_{2 m} T\left(I_{j}\right)$ , em que:

$\mathrm{Y}_{\mathrm{mj}}=$ resposta do genótipo ideal hipotético no ambiente j; $\mathrm{I}_{\mathrm{j}}=$ índice de ambiente codificado;

$\mathrm{T}\left(\mathrm{I}_{\mathrm{j}}\right)=\operatorname{se~} \mathrm{I}_{\mathrm{j}}<0$ e, $\mathrm{T}\left(\mathrm{I}_{\mathrm{j}}\right)=\mathrm{I}_{\mathrm{j}}-I_{+}$se $\mathrm{I}_{\mathrm{j}}>0$, sendo $I_{+}$a média dos índices I positivos;

$\mathrm{b}_{0 \mathrm{~m}}=$ valor fornecido para que a resposta ideal seja máxima para todos os locais;

$b_{1 m}$ e $b_{2 m}=$ coeficientes de regressão linear, cujos valores são atribuídos para que haja resposta o mais próximo possível do ideal

Assim sendo, para variáveis cujo interesse foi obter o máximo possível de desempenho estabeleceu-se $b_{0 m}$ igual ao máximo dos valores obtidos nos experimentos, $b_{1 \mathrm{~m}}=0,5$ que reflete baixa resposta aos ambientes desfavoráveis e $\mathrm{b}_{2 \mathrm{~m}}=1,0$ responsivo às condições favoráveis.

Para as variáveis acamamento, quebramento, ocorrência de pragas e doenças, cujo interesse é obter o menor valor possível, adotou-se valor de $b_{\text {om }}$ igual a 
zero ou, o menor valor obtido nos experimentos; $b_{1 \mathrm{~m}} \mathrm{e}$ $\mathrm{b}_{2 \mathrm{~m}}$ iguais a zero, desta forma, $\mathrm{Y}_{\mathrm{mj}}$ assumirá valor referencial baixo. Para altura de plantas, assumiu-se $b_{0 \mathrm{~m}}$ $=200, b_{1 m}$ e $b_{2 m}$ iguais a zero; assim, $Y_{m j}=200$, ou seja, o valor referencial para classificação dos genótipos é de $200 \mathrm{~cm}$ de altura, quer seja para ambiente favorável ou desfavorável.

A estimativa do parâmetro MAEC fica:

$$
P_{m i g}=\frac{\sum_{j=1}^{n}\left(Y_{i j}-Y_{m j}\right)^{2}}{2 n} \text {, para termos gerais, para o }
$$

cultivar i;

$$
P_{m i f}=\frac{\sum_{j=1}^{n}\left(Y_{i j}-Y_{m j}\right)^{2}}{2 f} \text {, para ambientes favoráveis, }
$$

para o cultivar $i$

$P_{m i d}=\frac{\sum_{j=1}^{n}\left(Y_{i j}-Y_{m j}\right)^{2}}{2 d}$, para ambientes desfavoráveis, para o cultivar i.

Sendo: $Y_{\mathrm{ij}}, \mathrm{n}, \mathrm{d}, \mathrm{f}, \mathrm{Y}_{\mathrm{mj}}$ definidos anteriormente.

\section{RESULTADOS E DISCUSSÃO}

Constataram-se efeitos significativos para ambientes, híbridos, interação híbridos x ambientes e desvios combinados (Tabela 1). Efeitos significativos para ambiente linear indicam presença de variações, significativas, nos ambientes para proporcionar alterações nas médias dos híbridos estudados e, para desvios combinados das regressões, falta de linearidade para, pelo menos, uma das equações ajustadas. No entanto, a regressão linear explicou mais que $80 \%$ para a maioria dos dados garantindo tomadas de decisão e conclusões sobre o desempenho dos híbridos estudados.

\section{Ensaio de safra normal 96/97}

Dos dezesseis híbridos testados em seis ambientes, apenas dois apresentaram adaptabilidade específica: D 170 para ambientes desfavoráveis $\left(\beta_{1 \mathrm{i}}<1\right.$ em nível de $1 \%$ de probabilidade) e o STAR para ambientes favoráveis $\left(\beta_{1 \mathrm{i}}>1\right.$ em nível de $5 \%$ de probabilidade) e, os demais, de adaptabilidade ampla segundo metodologia de EBERHART \& RUSSELL (1966). Apenas três híbridos (D 556, D 769 e Z 8501) apresentaram-se como instáveis aos níveis de 1\%,5\% e 5\% de probabilidade, respectivamente (Tabela 2).

A classificação de ambiente em favorável ou desfavorável é obtida considerando-se a média geral de todos os experimentos em que aquele com média maior que a média geral constitui-se como ambiente favorável e aquele com média menor que a média geral é o desfavorável. Nesse procedimento, se houver dois experimentos conduzidos no mesmo local e tipo de solo, um ao lado do outro, tomando-se todos os cuidados para se manter o máximo de uniformidade na condução dos experimentos, as médias de cada experimento certamente não serão as mesmas, de modo que sempre ter-se-á um deles como favorável e outro, desfavorável. Assim, a simples classificação de ambientes em favorável ou desfavorável não é suficiente para a discriminação da estabilidade e adaptabilidade genotípica, ou seja, pode não refletir a existência de divergência agro-ecológica (ambiental). No entanto, essa classificação dá uma idéia de como os genótipos se comportarão frente a certas variações ambientais e, desse modo vem sendo utilizada.

Uma maneira de se verificar a existência de divergência ambiental é através da análise de estratificação de ambientes que pode ser realizada do ponto de vista agro-ecológico, considerando-se o tipo de solo, topografia, altitude, temperatura, precipitação pluviométrica, etc. ou, do ponto de vista da genética quantitativa em que há possibilidades de agrupamento ambiental naqueles cuja interação genótipos $\mathrm{x}$ ambientes seja não significativa ou, ainda, por outras metodologias.

Neste ensaio, a classificação dos ambientes em favoráveis ou desfavoráveis não esteve relacionada somente à época de plantio, sendo muito importantes o manejo cultural dada ao experimento e à ocorrência de fatores imprevisíveis (temperatura, precipitação, pragas e doenças).

Apesar do híbrido STAR ser indicado para condições favoráveis segundo a metodologia de EBERHART \& RUSSELL (1966), ele não apresentou essa tendência tão significativa em LIN \& BINNS (1988) (Tabela 3). Por outro lado, a indicação do D 170 para condições desfavoráveis foi confirmada pelos dois métodos. Entre os híbridos com produtividade média superior á 4.000kg.ha-1, o D 766 apresentou melhor performance para condições favoráveis pela metodologia de LIN \& BINNS (1988), sendo discordante com a metodologia de EBERHART \& RUSSELL (1966). $\mathrm{O}$ híbrido $\mathrm{Z} 8452$, indicado para condições gerais segundo EBERHART \& RUSSELL (1966), passa a apresentar bom desempenho também para condições desfavoráveis em LIN \& BINNS (1988). Como pode ser observado, enquanto a metodologia de EBERHART \& RUSSELL (1966) aponta apenas dois genótipos para condições específicas, a metodologia de LIN \& BINNS (1988) apresenta quatro. Esses resultados confirmam 
Tabela 1 - Resultados das análises de variância conjuntas com a decomposição da soma de quadrados.

\begin{tabular}{lccc}
\hline Época & Fonte Variação & graus liberdade & Quadrado Médio \\
\hline Safra Normal 1996/97 & Ambientes (A) & 5 & $66.613 .217,8473$ \\
& Genótipos (G) & 15 & $2.351 .587,0151$ \\
& Interação AxG & 75 & $563.546,8216$ \\
& Amb./Genótipo & 80 & $4.691 .651,2607$ \\
& Ambiente Linear & 1 & $333.066 .089,2367$ \\
& Gx amb. linear & 15 & $418.829,0029$ \\
Desvios combinados & Resíduo & 64 & $562.243,3840$ \\
Safrinha/97 & Ambientes (A) & 780 & $336.655,2181$ \\
& Genótipos (G) & 7 & $58.330 .723,0408$ \\
& Interação AxG & 14 & $3.236 .637,2573$ \\
& Amb./Genótipo & 98 & $720.707,4203$ \\
& Ambiente Linear & 105 & $4.561 .375,1284$ \\
& Gx amb. linear & 1 & $408.315 .061,2853$ \\
& Desvios combinados & 14 & $406.415,9140$ \\
& Resíduo & 90 & $721.550,0488$ \\
& 224 & $400.178,5233$
\end{tabular}

$*, * *$ = significativo pelo teste $\mathrm{F}$ em níveis de 5 e $1 \%$ de probabilidade, respectivamente. $\mathrm{ns}=$ não significativo pelo teste $\mathrm{F}$.

que a metodologia de LIN \& BINNS (1988) seja mais discriminante.

Nas análises de estabilidade e adaptabilidade multivariada propostas por CARNEIRO (1998), baseado em LIN \& BINNS (1988), foram obtidos resultados discordantes das análises univariadas, indicando que outras variáveis apresentaram efeitos significativos na classificação dos genótipos; assim, por indução, chegase à conclusão de que este procedimento tem importante efeito complementar à metodologia univariada, pois, pode-se conhecer melhor os genótipos quando vários caracteres são considerados e seus ambientes caracterizados (Tabela 4).

O STAR se posicionou entre os últimos colocados para os ambientes favoráveis e desfavoráveis (Tabela 4). Essa mudança na classificação pode ser atribuída à alta ocorrência de plantas acamadas e quebradas (valores de $2,95 \%$ e $16,17 \%$, respectivamente) que pesaram na classificação multivariada do híbrido STAR. O MASTER praticamente apresentou a terceira posição para todas as condições embora sua produtividade média (3.905kg.ha-1) esteja abaixo da média geral (3.961kg.ha $\left.{ }^{-1}\right)$ o que indica a existência de alguma(s) característica(s) vantajosa(s), podendo-se destacar a baixa ocorrência de plantas acamadas (máximo de $0,65 \%$ das plantas).

Os caracteres, além de sua importância na classificação da performance genotípica, influenciam na respostas dos genótipos aos tipos de ambientes. Exemplificando essa afirmação, o híbrido D 170 que apresentou melhor desempenho para condições desfavoráveis tanto em EBERHART \& RUSSELL (1966) quanto para LIN \& BINNS (1988), ambos univariados, teve melhor desempenho para condições gerais quando outras variáveis foram consideradas. O Z 8501, no contexto multivariado, apresentou-se como mais indicado para condições favoráveis sendo que, nas análises univariadas foi indicado para condições gerais.

\section{Ensaio de safrinha/97}

Pelo método de EBERHART \& RUSSELL (1966), todos os quinze híbridos avaliados em oito ambientes seriam indicados para toda a rede por apresentar ampla adaptabilidade $(\beta=1)$, com exceção do híbrido Z 8202 que apresentou adidaptabilidade para alta tecnologia ( $\beta>1$, em nível de $5 \%$ de probabilidade). Cinco híbridos apresentaram-se como instáveis: D 766 (P<5\%), D 769 (P<5\%), Z 8452 (P<5\%), DENSUS $(\mathrm{P}<$ 1\%) e XL 9663 (P<5\%) e, os demais, estáveis (Tabela 2).

A classificação de ambientes no ensaio de safrinha/97 seguiu o mesmo padrão obtido no ensaio de safra normal, ou seja, não esteve somente relacionada com a época de plantio mas outros fatores também foram responsáveis para seu resultado final.

Dentre os híbridos mais produtivos e de adaptabilidade ampla, dada pela metodologia de EBERHART \& RUSSELL (1966), o D 657 e XL 345 apresentaram melhor performance para condições gerais e favorável, segundo procedimento de LIN \& BINNS

Ciência Rural, v. 34, n. 1, jan-fev, 2004. 
Tabela 2 - Resultados das análises da adaptabilidade e estabilidade segundo a metodologia de EBERHART \& RUSSELL (1966), nos ensaios de safra normal 1996/97 (6 ambientes) e safrinha/97 (8 ambientes) com as estimativas das médias de produtividade de grãos em kg.ha ${ }^{-1}$ $\left(\hat{\beta}_{0 i}\right)$, dos coeficientes de regressão $\left(\hat{\beta}_{1 i}\right)$, dos desvios de regressão $\left(\hat{\sigma}_{d i}^{2}\right)$ e dos coeficientes de determinação $\left(\mathrm{R}^{2}\right)$.

\begin{tabular}{|c|c|c|c|c|c|c|c|c|}
\hline F.V. & G.L. & Q.M. & $\begin{array}{l}\text { Média } \\
\left(\hat{\beta}_{0 i}\right)\end{array}$ & & & $=1)^{(1)}$ & $\begin{array}{c}\hat{\boldsymbol{\sigma}}_{d i}^{2} \\
\left(\mathrm{H} 0: \boldsymbol{\sigma}_{d i}^{2}=0\right)^{(2)}\end{array}$ & $\mathrm{R}^{2}(\%)$ \\
\hline \multicolumn{9}{|c|}{ Safra normal 1996/97 } \\
\hline D 657 & 4 & $542.712,2714$ & $4.737,80$ & 1,13 & NS & $68.685,68$ & NS & 92,40 \\
\hline D 556 & 4 & $1.338 .904,4409$ & $3.553,35$ & 1,11 & NS & $334.083,07$ & $* *$ & 82,69 \\
\hline D 766 & 4 & $668.926,5030$ & $4.011,46$ & 1,10 & NS & $110.757,09$ & NS & 90,44 \\
\hline D 170 & 4 & $15.589,6834$ & $3.728,05$ & 0,67 & $* *$ & $-107.021,84$ & NS & 99,33 \\
\hline D 769 & 4 & $946.018,2858$ & $3.578,73$ & 0,82 & NS & $203.121,02$ & $*$ & 78,70 \\
\hline Z 84E74 & 4 & $705.889,8900$ & $3.933,71$ & 1,04 & NS & $123.078,22$ & NS & 88,91 \\
\hline Z 8440 & 4 & $298.989,3087$ & $3.708,15$ & 1,00 & NS & $-12.555,30$ & NS & 94,58 \\
\hline Z 8452 & 4 & $163.019,5363$ & $4.090,00$ & 0,95 & NS & $-57.878,56$ & NS & 96,66 \\
\hline Z 8392 & 4 & $288.337,4192$ & $4.044,41$ & 0,89 & NS & $-16.105,93$ & NS & 93,42 \\
\hline Z 8501 & 4 & $1.085 .220,8643$ & $4.092,41$ & 1,08 & NS & $249.521,88$ & $*$ & 84,80 \\
\hline Z 84E90 & 4 & $705.479,8782$ & $3.537,40$ & 1,00 & NS & $122.941,55$ & NS & 87,97 \\
\hline SAVANA & 4 & $372.793,6925$ & $3.769,76$ & 0,91 & NS & $12.046,16$ & NS & 92,03 \\
\hline EXCELER & 4 & $364.989,2038$ & $3.686,32$ & 0,94 & NS & $9.444,66$ & NS & 92,66 \\
\hline MASTER & 4 & $443.697,0694$ & $3.905,69$ & 1,08 & NS & $35.680,62$ & NS & 93,16 \\
\hline AVANT & 4 & $420.049,6983$ & $4.545,62$ & 1,01 & NS & $27.798,16$ & NS & 92,68 \\
\hline \multirow[t]{2}{*}{ STAR } & 4 & $635.276,3983$ & $4.456,25$ & 1,28 & $*$ & $99.540,39$ & NS & 93,07 \\
\hline & & Média & $3.961,19$ & & & & & \\
\hline Resíduo & 180 & $336.655,2181$ & & & & & & \\
\hline \multicolumn{9}{|c|}{ Safrinha/97 } \\
\hline D-657 & 6 & $561.289,3406$ & $5.736,71$ & 0,95 & NS & $53.703,61$ & NS & 87,90 \\
\hline D-556 & 6 & $597.431,8243$ & $5.120,76$ & 0,89 & NS & $65.751,10$ & NS & 85,81 \\
\hline D-766 & 6 & $990.268,7957$ & $5.493,55$ & 1,08 & NS & $196.696,76$ & $*$ & 84,33 \\
\hline D-769 & 6 & $1.054 .187,4478$ & $4.731,44$ & 0,95 & NS & $218.002,97$ & $*$ & 79,62 \\
\hline Z-8202 & 6 & $676.389,2745$ & $5.175,73$ & 1,27 & $*$ & $92.070,25$ & NS & 91,52 \\
\hline Z-8452 & 6 & $920.054,6429$ & $5.225,08$ & 0,94 & NS & $173.292,04$ & $*$ & 81,35 \\
\hline Z-8392 & 6 & $186.136,8478$ & $5.499,93$ & 0,91 & NS & $-71.347,22$ & NS & 95,32 \\
\hline Z-8501 & 6 & $805.687,7481$ & $5.450,59$ & 1,06 & NS & $135.169,74$ & NS & 86,34 \\
\hline Z-8490 & 6 & $535.137,3174$ & $4.929,17$ & 1,19 & NS & $44.986,26$ & NS & 92,25 \\
\hline DENSUS & 6 & $1.243 .892,4853$ & $4.312,74$ & 0,89 & NS & $281.237,99$ & $* *$ & 74,31 \\
\hline EXCELER & 6 & $675.205,2317$ & $5.251,97$ & 1,02 & NS & $91.675,57$ & NS & 87,56 \\
\hline MASTER & 6 & $725.512,0168$ & $5.329,13$ & 0,87 & NS & $108.444,50$ & NS & 82,44 \\
\hline XL-360 & 6 & $355.452,0921$ & $5.035,56$ & 0,92 & NS & $-14.908,81$ & NS & 91,45 \\
\hline XL-345 & 6 & $506.647,8860$ & $5.531,12$ & 1,14 & NS & $35.489,79$ & NS & 92,12 \\
\hline \multirow[t]{2}{*}{ XL-9663 } & 6 & $989.957,7817$ & $4.847,01$ & 0,92 & NS & $196.593,09$ & $*$ & 79,40 \\
\hline & & Média & $5.178,03$ & & & & & \\
\hline Resíduo & 224 & 400178,5233 & & & & & & \\
\hline
\end{tabular}

(1) significância avaliada pelo teste t, a $1(* *)$ e $5 \%(*)$ de probabilidade,

(2) significância avaliada pelo teste F, a $1(* *)$ e $5 \%\left(^{*}\right)$ de probabilidade,

NS = não significativo.

(1988) (Tabela 3). O Z 8501 teve melhor desempenho para condições favoráveis, em oposição aos híbridos D 766 e MASTER, discordando dos resultados da metodologia de EBERHART \& RUSSELL (1966), em que estes foram de adaptabilidade ampla.
Considerando-se outras variáveis, alterações na classificação dos genótipos foram constatadas, confirmando existência de características de grande influência no valor final do genótipo que contribuem para melhor conhecimento das performances. Os híbridos Z 8452 eZ 8501 agora apresentaram os melhores 
Tabela 3 - Estimativas das médias de produtividade de grãos $\left(\mathrm{kg}_{\mathrm{g}} \mathrm{ha}^{-1}\right)$, dos parâmetros de estabilidade para condições gerais $\left(\mathrm{P}_{\mathrm{ig}}\right)$, favoráveis $\left(\mathrm{P}_{\mathrm{if}}\right)$ e desfavoráveis $\left(\mathrm{P}_{\mathrm{id}}\right)$, com suas respectivas classificações, segundo a metodologia de LIN \& BINNS (1988), modificada por CARNEIRO (1998), nos ensaios de safra normal 96/97 (6 ambientes) e safrinha/97 (8 ambientes), região sul do Estado de Mato Grosso.

\begin{tabular}{|c|c|c|c|c|c|c|c|}
\hline Híbridos & Média Geral & $P_{\text {ig }}$ & Classif. & $\mathrm{P}_{\text {if }}$ & Classif. & $P_{\text {id }}$ & Classif \\
\hline \multicolumn{8}{|c|}{ Safra normal 1996/97 } \\
\hline D 657 & $4.737,80$ & $15.489,37$ & 1 & $16.608,70$ & 1 & $14.370,03$ & 1 \\
\hline D 556 & $3.553,35$ & $1.021 .897,16$ & 14 & $805.247,04$ & 15 & $1.238 .547,29$ & 14 \\
\hline D 766 & $4.011,46$ & $486.626,59$ & 7 & $166.605,41$ & 4 & $806.647,78$ & 10 \\
\hline D 170 & $3.728,05$ & $781.852,72$ & 13 & $872.216,09$ & 16 & $691.489,35$ & 7 \\
\hline D 769 & $3.578,73$ & $1.078 .450,23$ & 16 & $617.891,84$ & 11 & $1.539 .008,63$ & 16 \\
\hline Z 84E74 & $3.933,71$ & $640.497,90$ & 9 & $401.486,28$ & 9 & $879.509,52$ & 12 \\
\hline Z 8440 & $3.708,15$ & $721.184,92$ & 11 & $659.966,75$ & 12 & $782.403,09$ & 9 \\
\hline Z 8452 & $4.090,00$ & $379.761,99$ & 4 & $337.892,60$ & 8 & $421.631,38$ & 4 \\
\hline Z 8392 & $4.044,41$ & $471.645,43$ & 6 & $285.744,09$ & 7 & $657.546,77$ & 6 \\
\hline Z 8501 & $4.092,41$ & $404.582,80$ & 5 & $281.982,08$ & 6 & $527.183,51$ & 5 \\
\hline Z 84E90 & $3.537,40$ & $1.060 .424,56$ & 15 & $698.179,62$ & 13 & $1.422 .669,51$ & 15 \\
\hline SAVANA & $3.769,76$ & $687.002,22$ & 10 & $561.487,30$ & 10 & $812.517,14$ & 11 \\
\hline EXCELER & $3.686,32$ & $771.145,53$ & 12 & $763.153,62$ & 14 & $779.137,44$ & 8 \\
\hline MASTER & $3.905,69$ & $596.707,99$ & 8 & $202.291,13$ & 5 & $991.124,86$ & 13 \\
\hline AVANT & $4.545,62$ & $76.533,58$ & 2 & $109.426,28$ & 3 & $43.640,88$ & 2 \\
\hline STAR & $4.456,25$ & $134.865,95$ & 3 & $23.634,61$ & 2 & $246.097,29$ & 3 \\
\hline Média & $3.961,19$ & & & & & & \\
\hline \multicolumn{8}{|c|}{ Safrinha/97 } \\
\hline D-657 & $5.736,71$ & $169.392,76$ & 1 & $84.959,13$ & 1 & $253.826,39$ & 3 \\
\hline D-556 & $5.120,76$ & $638.732,80$ & 9 & $737.599,88$ & 11 & $539.865,72$ & 9 \\
\hline D-766 & $5.493,55$ & $347.163,29$ & 4 & $448.281,81$ & 6 & $246.044,76$ & 2 \\
\hline D-769 & $4.731,44$ & $1.174 .747,58$ & 14 & $1.157 .254,77$ & 13 & $1.192 .240,38$ & 13 \\
\hline Z-8202 & $5.175,73$ & $658.576,17$ & 10 & $262.483,40$ & 5 & $1,054.668,94$ & 12 \\
\hline Z-8452 & $5.225,08$ & $565.420,97$ & 8 & $601.490,00$ & 9 & $529.351,93$ & 7 \\
\hline Z-8392 & $5.499,93$ & $255.335,27$ & 2 & $254.640,77$ & 4 & $256.029,77$ & 4 \\
\hline Z-8501 & $5.450,59$ & $375.058,04$ & 5 & $184.784,70$ & 2 & $565.331,38$ & 10 \\
\hline Z-8490 & $4.929,17$ & $886.125,05$ & 12 & $479.160,47$ & 7 & $1.293 .089,63$ & 14 \\
\hline DENSUS & $4.312,74$ & $1.918 .971,71$ & 15 & $1.483 .470,68$ & 15 & $2.354 .472,74$ & 15 \\
\hline EXCELER & $5.251,97$ & $503.658,88$ & 7 & $567.255,78$ & 8 & $440.061,98$ & 6 \\
\hline MASTER & $5.329,13$ & $456.122,07$ & 6 & $677.153,13$ & 10 & $235.091,01$ & 1 \\
\hline XL-360 & $5.035,56$ & $683.589,50$ & 11 & $835.784,21$ & 12 & $531.394,80$ & 8 \\
\hline XL-345 & $5.531,12$ & $292.624,98$ & 3 & $224.352,27$ & 3 & $360.897,68$ & 5 \\
\hline XL-9663 & $4.847,01$ & $1.002 .750,94$ & 13 & $1.360 .913,23$ & 14 & $644.588,65$ & 11 \\
\hline Média & $5.178,03$ & & & & & & \\
\hline
\end{tabular}

desempenhos, diferindo dos resultados das análises univariadas (Tabela 4). O D 657 continuou apresentando-se bom para condições gerais; no entanto, nos deparamos com resultado de difícil explicação. A análise multivariada indicou o EXCELER para condições favoráveis e desfavoráveis, o que não faz sentido pois é incoerente uma metodologia indicar um genótipo para condições favoráveis e desfavoráveis e não o ser para condições gerais. Da mesma forma, se um genótipo vai bem para condições gerais, ela deveria apresentar bom desempenho para condições desfavoráveis e favoráveis também. Ainda não há explicação para tais resultados.

\section{CONCLUSÕES}

A partir dos resultados obtidos, pode-se concluir que a metodologia de LIN \& BINNS (1988) modificada por CARNEIRO (1998) foi mais discriminante do que EBERHART \& RUSSELL (1966) e por isso, mais eficiente e mais indicada para análises de estabilidade e adaptabilidade. A análise multivariada permitiu conhecer melhor a performance

Ciência Rural, v. 34, n. 1, jan-fev, 2004. 
Tabela 4 - Estimativas dos parâmetros de estabilidade multivariada para condições geral $\left(\mathrm{P}_{\text {mig }}\right)$, favorável $\left(\mathrm{P}_{\text {mif }}\right)$, desfavorável ( $\left.\mathrm{P}_{\text {mid }}\right)$ com suas classificações, segundo a metodologia de LIN \& BINNS (1988), modificada por CARNEIRO (1998), nos ensaios de safra normal 96/97 (6 ambientes) e safrinha/97 (8 ambientes), região sul do Estado de Mato Grosso.

\begin{tabular}{|c|c|c|c|c|c|c|}
\hline Híbridos & $\mathrm{P}_{\mathrm{mig}}$ & Classif. & $\mathrm{P}_{\mathrm{mif}}$ & Classif. & $\mathrm{P}_{\text {mid }}$ & Classif. \\
\hline \multicolumn{7}{|c|}{ Safra normal 1996/97 } \\
\hline D-657 & 0,9208 & 1 & 1,2406 & 2 & 0,9027 & 1 \\
\hline D-556 & 1,3345 & 7 & 1,4361 & 4 & 1,2014 & 4 \\
\hline D-766 & 1,3292 & 6 & 1,4379 & 5 & 1,2032 & 5 \\
\hline D-170 & 1,2091 & 4 & 1,6010 & 12 & 1,4508 & 13 \\
\hline D-769 & 1,3394 & 8 & 1,4779 & 7 & 1,2108 & 6 \\
\hline Z-84E74 & 1,7058 & 16 & 1,5917 & 11 & 1,2537 & 9 \\
\hline Z-8440 & 1,5564 & 15 & 1,9430 & 16 & 1,6050 & 15 \\
\hline Z-8452 & 1,2358 & 5 & 1,4888 & 8 & 1,2218 & 7 \\
\hline Z-8392 & 1,3892 & 12 & 1,5762 & 10 & 1,2747 & 10 \\
\hline Z-8501 & 1,4819 & 14 & 1,4722 & 6 & 1,2376 & 8 \\
\hline Z-84E90 & 1,3608 & 10 & 1,5379 & 9 & 1,3032 & 12 \\
\hline SAVANA & 1,4584 & 13 & 1,6014 & 13 & 1,2999 & 11 \\
\hline EXCELER & 1,3876 & 11 & 1,7155 & 14 & 1,5392 & 14 \\
\hline MASTER & 1,1757 & 2 & 1,3042 & 3 & 1,0695 & 3 \\
\hline AVANT & 1,1980 & 3 & 1,1921 & 1 & 1,0659 & 2 \\
\hline STAR & 1,3600 & 9 & 1,8577 & 15 & 1,6230 & 16 \\
\hline \multicolumn{7}{|c|}{ Safrinha/97 } \\
\hline D-657 & 0,9828 & 3 & 1,4889 & 9 & 1,1130 & 8 \\
\hline D-556 & 1,0537 & 5 & 1,3886 & 8 & 1,0366 & 6 \\
\hline D-766 & 1,0360 & 4 & 1,0285 & 4 & 0,8032 & 4 \\
\hline D-769 & 1,2716 & 9 & 1,7120 & 11 & 1,3600 & 10 \\
\hline Z-8202 & 1,7142 & 13 & 1,8899 & 13 & 1,6646 & 13 \\
\hline Z-8452 & 0,8809 & 1 & 0,8312 & 2 & 0,6243 & 2 \\
\hline Z-8392 & 1,4481 & 11 & 1,7919 & 12 & 1,5667 & 12 \\
\hline Z-8501 & 0,9312 & 2 & 0,7693 & 1 & 0,4842 & 1 \\
\hline Z-8490 & 1,7329 & 14 & 1,9484 & 14 & 1,7231 & 15 \\
\hline DENSUS & 1,8640 & 15 & 1,9921 & 15 & 1,7070 & 14 \\
\hline EXCELER & 1,0787 & 6 & 1,0262 & 3 & 0,8009 & 3 \\
\hline MASTER & 1,3249 & 10 & 1,6363 & 10 & 1,3919 & 11 \\
\hline XL-360 & 1,7125 & 12 & 1,3785 & 7 & 1,1341 & 9 \\
\hline XL-345 & 1,1696 & 8 & 1,1760 & 5 & 0,9867 & 5 \\
\hline XL-9663 & 1,1435 & 7 & 1,3073 & 6 & 1,0429 & 7 \\
\hline
\end{tabular}

dos genótipos, constituindo-se em importante ferramenta complementar na indicação de genótipos de acordo com seu desempenho.

\section{REFERÊNCIAS BIBLIOGRÁFICAS}

CARNEIRO, P.C.S. Novas metodologias de análise da adaptabilidade e estabilidade de comportamento. 1998. 168f. Tese (Doutorado) - Curso de Pós-graduação em Genética e Melhoramento, Universidade Federal de Viçosa.

CRUZ, C.D.; REGAZZI, A.J. Modelos biométricos aplicados ao melhoramento genético. 2. ed. Viçosa, MG: UFV, 1997. 390p.

CRUZ, C.D.; TORRES, R.A. de; VENCOVSKY, R. An alternative approach to the stability analysis proposed by
Silva e Barreto. Revista Brasileira de Genética, Ribeirão Preto, v.12, p.567-580, 1989.

EBERHART, S.A.; RUSSELL, W.A. Stability parameters for comparing varieties. Crop Science, v.6, n.1, p.36-40, 1966.

EMBRAPA - Empresa Brasileira de Pesquisa Agropecuária. Recomendações técnicas para a cultura da soja - Região Centro-Oeste. Brasília : EMBRAPA-SPI, 1993. p.14-20.

LIN, C.S.; BINNS, M.R. A superiority measure of cultivar performance for cultivar $\mathrm{x}$ location data. Canadian Journal of Plant Science, v.68, n.3, p.193-198, 1988.

MONTEIRO, J.A. et al. Produção de milho no Brasil Realidade e Perspectivas. In: CONGRESSO NACIONAL DE MILHO E SORGO, 19., 1992, Porto Alegre, RS. Conferências... Porto Alegre : SOCIEDADE BRASILEIRA DE MILHO E SORGO, 1992. p.81-126. 